Assessment of welfare and brachycephalic obstructive airway syndrome signs in young, breeding age French Bulldogs and Pugs, using owner questionnaire, physical examination and walk tests

\title{
Aromaa, M.
}

2019-08

Aromaa , M , Lilja-Maula , L \& Rajamäki , M M 2019 , ' Assessment of welfare and brachycephalic obstructive airway syndrome signs in young, breeding age French Bulldogs and Pugs, using owner questionnaire, physical examination and walk tests ' , Animal Welfare , vol. 28 , no. 3 , pp. 287-298 . https://doi.org/10.7120/09627286.28.3.287

other

publishedVersion

Downloaded from Helda, University of Helsinki institutional repository.

This is an electronic reprint of the original article.

This reprint may differ from the original in pagination and typographic detail.

Please cite the original version. 


\title{
Assessment of welfare and brachycephalic obstructive airway syndrome signs in young, breeding age French Bulldogs and Pugs, using owner questionnaire, physical examination and walk tests
}

\author{
M Aromaa*, L Lilja-Maula and MM Rajamäki
}

Department of Equine and Small Animal Medicine, Faculty of Veterinary Medicine, University of Helsinki, PO Box 57, 000 I4 Helsinki, Finland

* Contact for correspondence: mimma.aromaa@helsinki.fi

\begin{abstract}
Brachycephalic obstructive airway syndrome (BOAS) is a major welfare problem in short-nosed breeds, such as the French Bulldog and Pug. In addition to respiratory difficulties, exercise intolerance and impaired recovery are major signs of BOAS. To select healthier breeding animals, exercise tolerance tests, such as the I,000-m walk test, are already used in several countries for brachycephalic dogs, although evidence supporting their use is still scarce. The aims of this study were to assess the daily welfare of young, breeding-age French Bulldogs $(n=44)$ and Pugs $(n=5 I)$ using an owner questionnaire, and to evaluate 6-min walk test (6MWT) and I,000-m walk test usability for differentiation between non- or mildly BOAS-affected dogs and more severely affected dogs. Only four out of 95 French Bulldog and Pug owners reported that the BOAS signs limited the daily activities of their dogs. However, according to the physical, examination-based veterinary BOAS grading, 31/95 of the dogs had moderate to severe BOAS signs. In both breeds, the more severely affected dogs performed both exercise tests more poorly than those with no or mild BOAS signs. The longer exercise, namely the I,000-m test, seemed slightly better able at differentiating between affected dogs and less affected ones. The results of this study further support the use of exercise tests as an important part of the breeding selection in French Bulldogs and Pugs. By influencing the breed standards set by Kennel Clubs and by using breeding selection tools, the harmful impacts of brachycephaly can be diminished.
\end{abstract}

Keywords: animal welfare, brachycephalic obstructive airway syndrome, dog, French Bulldog, Pug, walk test

\section{Introduction}

The enormous growth in popularity of brachycephalic breeds (Emmerson 2014; Kennel Club 2017, 2018), such as the French Bulldog, Pug and English Bulldog, and the high prevalence of several health problems occurring in these breeds has created a major welfare issue. The breathing difficulties, known as brachycephalic obstructive airway syndrome (BOAS), are directly linked to the short anatomical structure of the skull (Oechtering 2010; Emmerson 2014). BOAS includes a variety of clinical signs, such as loud upper respiratory sounds, sniffing, respiratory distress, sleeping disorders, exercise and heat intolerance, cyanosis, gastrointestinal signs and, in severe cases, fainting or even death (Hendricks 2004; Poncet et al 2005, Riecks et al 2007; Roedler et al 2013).

As BOAS is related to congenitally flattened facial and skull anatomy, the key to healthier brachycephalic dogs lies in the breed standards set by kennel clubs and the breeding choices made by breeders (Oechtering 2010; Packer \& Tivers 2015). By avoiding extreme facial structures and selecting dogs without clinically relevant BOAS signs, the harmful consequences of brachycephaly might be reduced and the welfare of the offspring increased. However, not all owners and breeders recognise the signs of BOAS or interpret them as normal for the breed (Packer et al 2012; Roedler et al 2013). Therefore, an objective, non-invasive and economical test to guide responsible breeding choices is urgently needed.

An easy way to objectively predict probability of BOAS affectedness on a larger scale would be a soft tape measurement of conformational risk factors, such as the craniofacial ratio (CFR) (Packer et al 2015). However, since the muzzle length is extremely short and its variation within brachycephalic breeds is minimal, the CFR has not been proven to reliably predict the BOAS affectedness in these dogs (Liu et al 2017). In terms of other conformational risk factors, visually assessed stenosis of the nostrils has been found to be a strong predictor of BOAS in all three of the most popular brachycephalic breeds, the French Bulldog, Pug and English Bulldog, and neck girth ratio in male English Bulldogs (Liu et al 2017). However, not all individuals with a low CRF or stenotic nostrils suffer from BOAS (Packer et al 2015; Liu et al 2017). Moreover, the respiratory function measurement with whole-body barometric plethysmography (WBBP) has been demonstrated to be an objective and reliable method of 
evaluating the severity of BOAS (Liu et al 2015, 2016), but its use is currently limited to research settings.

As exercise intolerance and delayed recovery are major consequences of BOAS, sub-maximal exercise tests, such as the 6-min walk test (6MWT) and 1,000-m walk test, offer an objective way to assess the severity of BOAS. The 6MWT has been shown to be an easy and replicable test to screen exercise tolerance in dogs with induced heart disease, obesity and various lung diseases (Boddy et al 2004; Swimmer \& Rozanski 2011; Lilja-Maula et al 2014; Manens et al 2014). Although studies regarding 1,000-m test usability were lacking, this test with set time and recovery limits had already been in use as a breeding selection test in Germany since 2009 for Pugs (Bartels et al 2015), and in The Netherlands since 2014 for English Bulldogs (Dutch Kennel Club 2014). Subsequently, we demonstrated that English Bulldogs with more severe BOAS walked a shorter distance during the 6MWT, and more slowly during the $1,000-\mathrm{m}$ test, and that their recovery from exercise took longer than those with only mild signs of BOAS (LiljaMaula et al 2017). However, differences in anatomy can influence exercise performance and BOAS signs, and therefore the results for English Bulldogs cannot be directly extrapolated to other brachycephalic breeds. For this reason, the optimal exercise and recovery limits for any walk tests in different brachycephalic breeds are still needed.

The aims of this study were to evaluate 6MWT and $1,000-\mathrm{m}$ walk test usability in young, breeding age French Bulldogs and Pugs, and to examine the relationship between the veterinary assessed severity of BOAS and the exercise test results. Additionally, the presence of BOAS signs and their impact on the daily life of these young, considered healthy, French Bulldogs and Pugs were assessed using an owner questionnaire.

\section{Materials and methods}

\section{Ethical approval}

All animals were privately owned, prospectively recruited pet dogs, the owners of which had signed an informed consent form. The study protocol was approved by the Committee of Experimental Animals of Southern Finland (no: ESAVI/11519/04.10.07/2014).

\section{Study animals and protocol}

The study was performed at the Veterinary Teaching Hospital, University of Helsinki, Finland between November 2015 and May 2017. The inclusion criteria for brachycephalic dogs were purebred French Bulldog or Pug, aged between one and five years, with no history of airway surgery or treatment due to BOAS. Healthy dogs in the same age range and of small- or medium-sized $(5-15 \mathrm{~kg})$ breeds were recruited as control dogs.

During the study visit, an owner questionnaire regarding BOAS signs was completed, a physical examination and exercise tests were performed, and blood samples for haematology and biochemistry were taken to rule out systemic diseases.

\section{Daily welfare evaluation}

Evaluation of owner-perceived daily welfare related to BOAS signs was carried out by owner interview. The questions (modified from Roedler et al 2013) included general assessment of dogs' overall well-being (ie does your dog have any illness or problem affecting its daily welfare), exercise and heat tolerance, eating and sleeping habits and breathing.

\section{Grading of BOAS signs}

The physical examination was performed, and the severity of BOAS assessed by evaluating audible upper respiratory noises, the use of accessory respiratory muscles and the colour of the mucous membranes before and after the exercise test. All of the dogs were graded as having no (grade 0), mild (grade 1), moderate (grade 2) or severe (grade 3) BOAS signs according to a functional grading system modified from Liu et al (2015) and presented in Lilja-Maula et al (2017). Based on the grading, the dogs were further classified into either a BOAS- group (grades 0 and 1) or a BOAS+ group (grades 2 and 3), as presented in Liu et al (2015). Stenosis of the nares was visually graded as open, mild, moderate, or severe as previously described in Packer et al (2015) and Liu et al (2016).

\section{Exercise test}

Respiratory rate and type, upper airway sounds, heart rate and body temperature were evaluated prior to, between the $6 \mathrm{MWT}$ and the $1,000-\mathrm{m}$ test, and after walking. As previously described (Lilja-Maula et al 2017), the dogs were walked along a quiet, $60-\mathrm{m}$ straight, air-conditioned $\left(21-22^{\circ} \mathrm{C}\right)$ corridor on a leash at their own pace for $6 \mathrm{~min}$. The distance walked in $6 \mathrm{~min}$ was recorded, the dogs continued walking until 1,000 $\mathrm{m}$ was reached, and the time excluding the break was recorded again. Dogs were encouraged to walk or trot as briskly as possible; no cantering or galloping was allowed. The dogs' recovery time (heart and respiratory rate and body temperature) was monitored at 5-min intervals until the animal had recovered to the pre-walking status. Exercise tests were not carried out or were discontinued if the animal had respiratory distress or a body temperature above $39.5^{\circ} \mathrm{C}$.

\section{Statistical analysis}

Descriptive statistics are presented as a mean $( \pm$ SD) for continuous normally distributed variables or as a median and range for non-continuous or non-normally distributed variables. For pre- and post-exercise body temperature comparison in French Bulldogs, Pugs and controls, a paired $t$-test was used. To compare the 6MWT distance (6MWD) and $1,000-\mathrm{m}$ time in the BOAS+ and BOAS- groups, an independent sample $t$-test without the assumption of equal variances was used, while the Mann-Whitney $U$ test was chosen to compare recovery time intervals and stenosis of the nostrils in both groups.

The diagnostic ability of the two walk tests (6MWT and $1,000-\mathrm{m})$ in diagnosing BOAS was investigated using receiver-operating characteristic (ROC) curves. The optimal cut-off value for the test, namely distance or time, was defined 
as the point where the sum of sensitivity and specificity of the test reaches its maximum value. In addition, a suggestion for the normal range for the walk test results was defined as the mean $( \pm 2 \mathrm{SD})$ of the control dogs. The association between BOAS severity and the 6MWD and 1,000-m test time was analysed with an analysis of variance model (ANOVA). The model included the BOAS severity class $(0,1,2,3)$ as the fixed effect, and age and weight as covariates. The within BOAS-class estimates and pair-wise differences between the BOAS classes were calculated from the model using least square means (LSM) and confidence intervals (CI). No corrections for multiplicity were made. The normality of the 6MWT and 1,000-m test values was assessed using the Shapiro-Wilk test for normality and normal QQ plots. Statistical analyses were conducted using SAS ${ }^{\circ}$ System for Windows, version 9.3 (SAS Institute Inc, Cary, NC, USA) and R for Windows ${ }^{\circledR}$, version 3.5.0 (R Foundation for Statistical Computing, Vienna, Austria), and the GraphPad Prism Mac 7.0 d $®$. $P$-values $<$ 0.05 were considered statistically significant.

\section{Results}

\section{Dogs}

The brachycephalic population consisted of 44 French Bulldogs and 51 Pugs. The 21 control dogs represented nine breeds: six were Border Terriers, two Cairn Terriers, seven Miniature Poodles, and one of each of the following — Jack Russell Terrier, West Highland White Terrier, Cavalier King Charles Spaniel, standard Dachshund, Mudi, and Cocker Spaniel. The results of owner interviews, physical examination and walk tests for $9 / 21$ control dogs have been reported previously (Lilja-Maula et al 2017). The demographics of all of the dogs are presented in Table 1.

\section{Daily welfare questionnaire}

Owners reported that 37/44 (84\%) French Bulldogs and 47/51 (92\%) Pugs did not have any health issues affecting their daily lives, although in the case of 16/44 (36\%) French Bulldogs and $17 / 51$ (33\%) Pugs, owners reported that their dogs had exercise intolerance. Two French Bulldog and two Pug owners reported that respiratory signs were limiting daily activity. Five of the French Bulldogs and two of the Pugs had persistent dermatological problems and one French Bulldog and one Pug had had intermittent orthopaedic issues affecting their daily lives. In addition, four French Bulldog owners stated that their dog had experienced a syncopal episode.

Warm outdoor temperatures affected dogs' daily exercise habits and recovery (Figures 1 and 2). Gastrointestinal signs, vomiting or regurgitation were reported to occur weekly in $3 / 44$ (7\%) French Bulldogs, occasionally in 13/44 (30\%) French Bulldogs, and in 7/51 (14\%) Pugs. Owners had noticed a loud upper respiratory noise in 35/44 (80\%) French Bulldogs and 41/50 (82\%) Pugs. The owner-reported presence of increased upper respiratory sounds in different situations is provided in Table 2. Owners reported that $4 / 44$ (9\%) French Bulldogs and 2/49 (4\%) Pugs sometimes slept with their chin elevated, and 2/44 (5\%) French Bulldog and $1 / 49$ (2\%) Pug owners had noticed that their dog often slept with an item of some sort in their mouth.
Table I Demographic data for the brachycephalic and control dogs.

\begin{tabular}{|c|c|c|c|}
\hline Factor & $\begin{array}{l}\text { French Bulldogs } \\
(n=44)\end{array}$ & $\begin{array}{l}\text { Pugs } \\
(n=5 I)\end{array}$ & $\begin{array}{l}\text { Control dogs } \\
(n=2 I)\end{array}$ \\
\hline \multicolumn{4}{|l|}{ Gender } \\
\hline Female & 25 & 31 & II \\
\hline Male & 19 & 20 & 10 \\
\hline \multicolumn{4}{|l|}{ Age (years) } \\
\hline Median/range & $2.5(I-5.5)$ & $3.0(I-5)$ & $3.0(1.5-5)$ \\
\hline \multicolumn{4}{|l|}{ Weight (kg) } \\
\hline Mean $( \pm S D)$ & $12.1( \pm 1.6)$ & $8.7( \pm 1.4)$ & $8.8( \pm 2.0)$ \\
\hline \multicolumn{4}{|l|}{$B C S(I-5)$} \\
\hline Median/range & $3(3-4)$ & $3(2-4)$ & $3(2-3)$ \\
\hline
\end{tabular}

Owners reported that 19/21 (90\%) of the control dogs did not have any health issues affecting their daily lives. Two dogs had persistent dermatological problems. No exercise intolerance or syncopal episodes were reported in any of the dogs. Warm weather $\left(>19^{\circ} \mathrm{C}\right)$ did not have an effect on the dogs' physical performance. All of the control dogs could walk for over $60 \mathrm{~min}$, and all recovered within $5 \mathrm{~min}$. Vomiting or regurgitation were seen occasionally in $8 / 21$ (38\%) control dogs. No loud upper respiratory noises or sleep disturbances were reported.

\section{Physical examination findings}

During the physical examination, upper respiratory sounds were evaluated at rest. No upper respiratory sounds at all were noted in 24/44 (55\%) French Bulldogs and 21/51 (41\%) Pugs. Mild upper respiratory sounds were assessed in 16/44 (36\%) French Bulldogs and 24/51 (47\%) Pugs, and moderate sounds in 3/44 (7\%) French Bulldogs and 6/51 $(12 \%)$ Pugs. Severe upper respiratory sounds were noted in one French Bulldog, but not in any Pugs. The French Bulldog with severe upper respiratory sounds also had respiratory distress and an abnormal respiratory pattern and, for this reason, a reliable evaluation of lower respiratory sounds was not possible. In all of the other dogs, the respiratory pattern, lower respiratory sounds and colour of the mucous membranes were normal. The physical examinations were otherwise normal.

Blood samples were taken from 42/44 French Bulldogs, 49/51 Pugs and 11/21 control dogs. No notable changes were detected in the haematological analyses. Three French Bulldogs had a remarkably high (> 3 times upper limit), two had a moderate (2-3 times upper limit) and six had a mild $(<2$ times upper limit) increase in alanine transaminase (ALAT). One French Bulldog had a mild increase in alkaline phosphatase (AFOS). One Pug had a moderate and two a mild increase in ALAT. All of the dogs with increased liver enzymes were clinically asymptomatic.

The physical examinations were normal in all of the control dogs. Two had a mild increase in AFOS but no other remarkable changes were noted in the haematological or biochemical analyses. 
Figure I

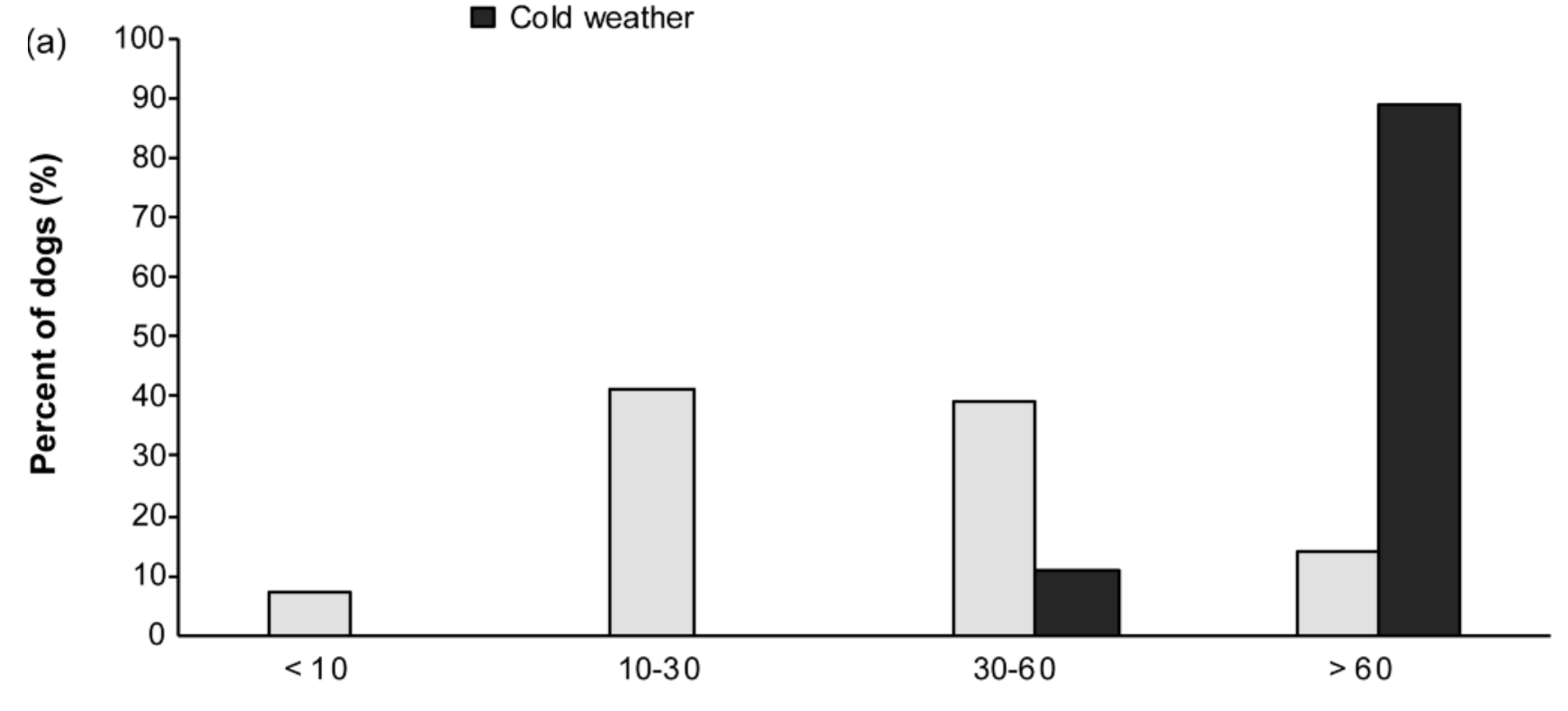

\section{Length of time $(\mathrm{min})$}

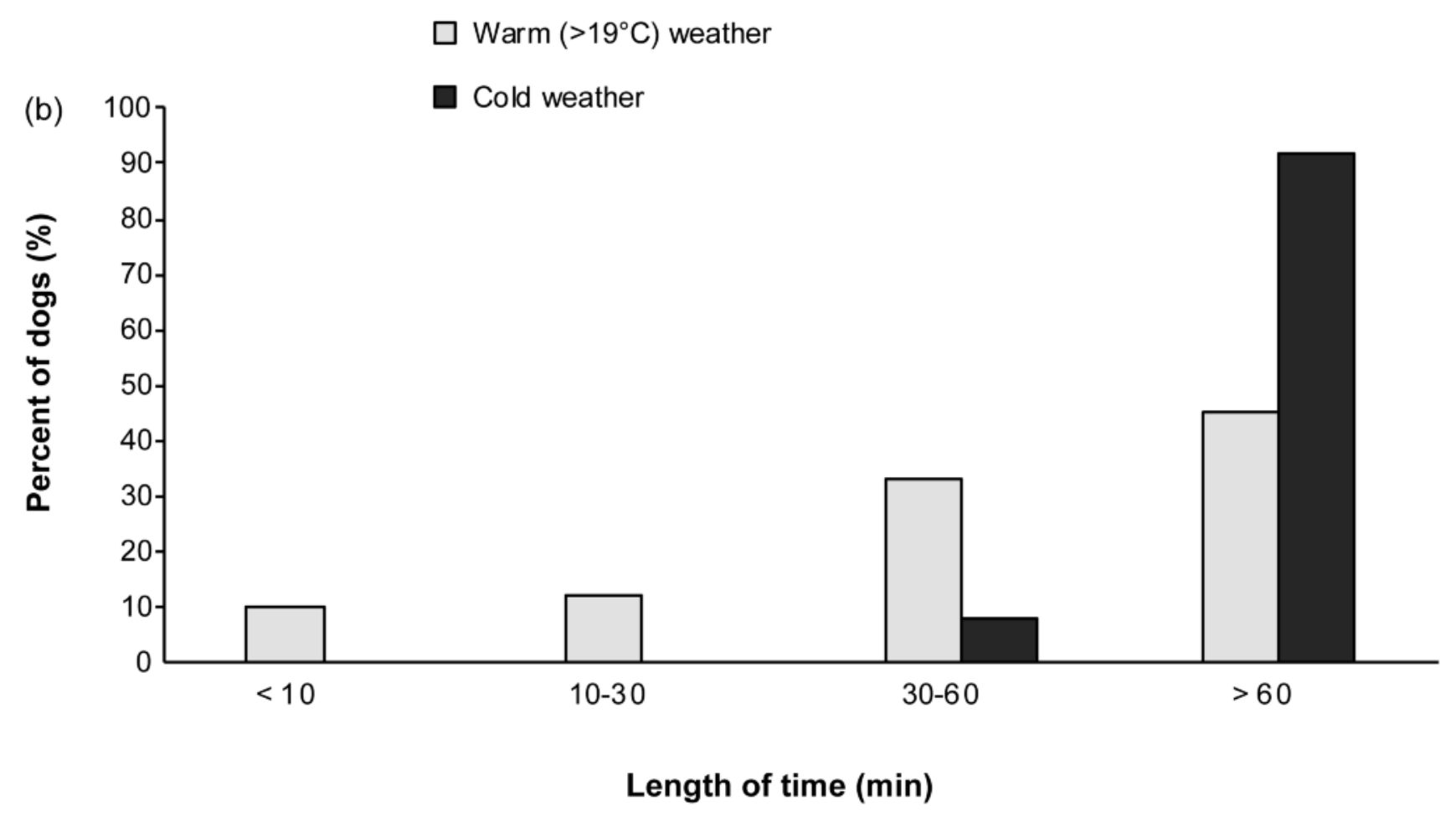

Responses of owners of (a) French bulldogs $(n=44)$ and $(b)$ Pugs $(n=5 I)$ to the daily welfare question, 'For how long a walk you go with your dog during warmer $\left(>19 \mathrm{C}^{\circ}\right)$ weather/colder weather?' 
Figure 2
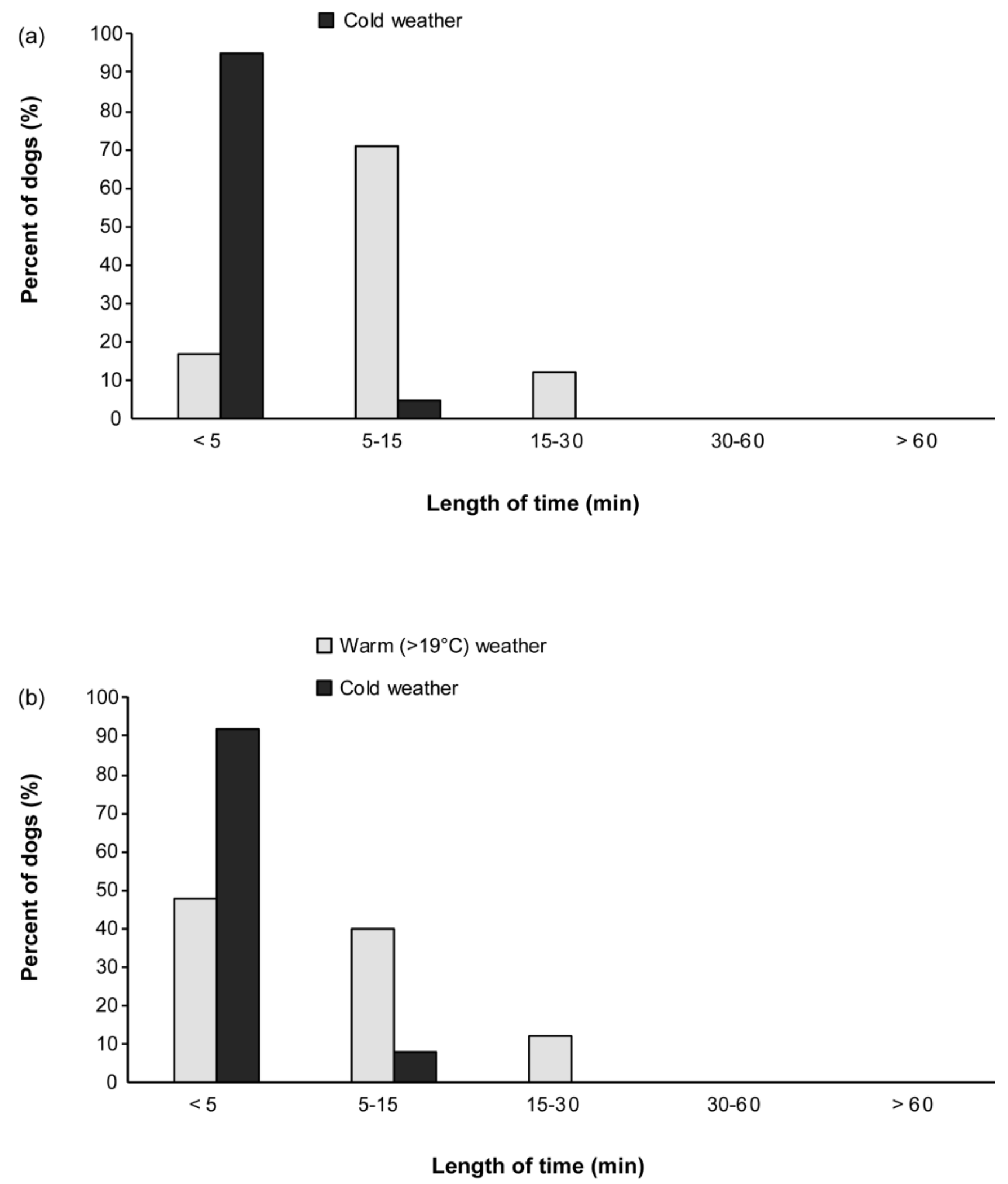

Responses of owners of (a) French bulldogs $(n=4 I)$ and (b) Pugs $(n=50)$ to the daily welfare question, 'How long does your dog need to recover after exercise during warmer $\left(>19 C^{\circ}\right)$ weather/colder weather?' 
Table 2 Owner-reported presence of increased upper respiratory sounds in French Bulldogs and Pugs in different situations.

\begin{tabular}{lll}
\hline Situation & $\begin{array}{l}\text { French Bulldogs } \\
(\mathbf{n}=35)\end{array}$ & $\begin{array}{l}\text { Pugs } \\
(\mathbf{n}=4 \mathrm{I})\end{array}$ \\
\hline When sleeping & $26(74 \%)$ & $29(70 \%)$ \\
During excercise/excitement & $25(71 \%)$ & $31(75 \%)$ \\
During warm weather $\left(>19^{\circ} \mathrm{C}\right)$ & $12(34 \%)$ & $18(45 \%)$ \\
At rest & $0(0 \%)$ & $0(0 \%)$ \\
\hline
\end{tabular}

Table 3 Distribution of veterinary-assessed severity of brachycephalic obstructive airway syndrome signs in French Bulldogs and Pugs.

\begin{tabular}{lcccc}
\hline Breed & $\begin{array}{c}\text { Grade 0 } \\
\text { BOAS- }\end{array}$ & $\begin{array}{c}\text { Grade I } \\
\text { BOAde 2 }\end{array}$ & $\begin{array}{c}\text { Grade 3 } \\
\text { BOAS+ }\end{array}$ \\
\hline $\begin{array}{l}\text { French } \\
\text { Bulldogs } \\
(n=44)\end{array}$ & 6 & 25 & 9 & 4 \\
$\begin{array}{l}\text { Pugs } \\
(n=51)\end{array}$ & 6 & $31(70 \%)$ & & $13(30 \%)$ \\
& & $33(65 \%)$ & 10 & 8 \\
\hline
\end{tabular}

BOAS: brachycephalic obstructive airway syndrome (grade $0=$ no, grade $1=$ mild, grade $2=$ moderate, grade 3 = severe signs);

BOAS $-=$ no or mild signs; $\mathrm{BOAS}+=$ moderate or severe signs.

\section{Severity of brachycephalic obstructive airway syndrome}

The veterinarian-assessed severity of BOAS is presented in Table 3. All of the control dogs were graded as having no signs comparable to BOAS.

None of the French Bulldogs or Pugs had open nostrils. Mild stenosis was noted in 6/44 (14\%), moderate stenosis in $26 / 44(59 \%)$ and severe stenosis in 12/44 (27\%) French Bulldogs. Similarly, the stenosis was noted as mild in 10/51 $(20 \%)$, moderate in $31 / 51(61 \%)$ and severe in $10 / 51(20 \%)$ Pugs. The difference in the level of stenosis was not statistically significant between the BOAS- and BOAS+ groups in French Bulldogs $(P=0.61)$ or in Pugs $(P=0.82)$.

\section{Exercise test}

When it came to the exercise test, 39/44 French Bulldogs, 48/51 Pugs and 19/21 control dogs performed the 6MWT, and 36/44 French Bulldogs, 47/51 Pugs and 19/21 control dogs performed the 1,000-m test. Two brachycephalic dogs were considered ineligible for either exercise test: one French Bulldog because of respiratory distress at rest, and one Pug because of orthopaedic problems. Three French Bulldogs did not proceed to the $1,000-\mathrm{m}$ test after the $6 \mathrm{MWT}$ due to an increase in body temperature over $39.5^{\circ} \mathrm{C}$, and one Pug because of exhaustion. Four French Bulldogs, two Pugs and two control dogs refused to walk properly on account of motivation problems during the exercise tests; these dogs were duly withdrawn from the analysis. The exercise test results are presented in Table 4. Based on the results of the control dogs and defined as mean ( $\pm 2 \mathrm{SD})$, the minimum normal value for 6MWT distance was $530.2 \mathrm{~m}$ and the maximum value for the 1,000-m test time was $10.43 \mathrm{~min}$.

The ability of the 6MWT distance and 1,000-m test time to distinguish BOAS- dogs from $\mathrm{BOAS}+$ dogs is represented by ROC curves (Figures 3 and 4). The associations between BOAS severity and the 6MWD and 1,000-m test time is presented in Table 5.

\section{Body temperature}

The pre- and post-exercise (1,000-m) test body temperatures are presented in Table 6.

The difference in the change of body temperature regarding the pre- versus the post-1,000-m test was statistically significant between the French Bulldogs and the control dogs $\left(P=0.001\right.$; a mean of $0.60^{\circ} \mathrm{C}$ increase for French Bulldogs; $95 \%$ CI 0.48 to $0.73^{\circ} \mathrm{C}$; a mean of $0.22^{\circ} \mathrm{C}$ increase for control dogs; $95 \%$ CI 0.03 to $0.40^{\circ} \mathrm{C}$ ), and between the Pugs and the control dogs $\left(P=0.003\right.$; a mean of $0.56^{\circ} \mathrm{C}$ increase for Pugs; $95 \%$ CI 0.43 to $\left.0.68^{\circ} \mathrm{C}\right)$.

\section{Discussion}

In the present study we have further demonstrated that exercise tests can be used when assessing the severity of BOAS signs in two popular brachycephalic breeds, the French Bulldog and the Pug. As the clinical severity of BOAS tends, mainly, to be subjectively assessed, the walk tests provide an easy and objective addition to breeding animal selection. In addition, we have evaluated by means of owner questionnaires the extent to which BOAS signs interfere in the daily lives of these young, breeding age dogs.

Daily welfare was assessed using questions from the previously developed and validated pre-BOAS surgery questionnaire (Roedler et al 2013). When comparing the questionnaire results from Roedler et al (2013) with our study, it is clear that BOAS signs are not as common in our study population as in Roedler et al (2013), since none of the dogs in our study were presented for BOAS surgery. This difference is reflected, in particular, in the findings concerning exercise intolerance, respiratory noise and sleeping problems. Exercise intolerance was reported in onethird of the dogs in our study, as it was noted in the majority $(88 \%)$ of dogs suffering from severe brachycephaly (Roedler et al 2013). None of the dogs in our study needed $30 \mathrm{~min}$ or more to recover from exercise during the summer and only $8 \%$ were unable to walk for more than 10 min during warm weather, whereas half of the dogs with severe BOAS in the study by Roedler et al (2013) needed $30 \mathrm{~min}$ or longer to recover, and one-third were able to walk for a maximum of $10 \mathrm{~min}$ in warmer weather. Owner-reported loud upper respiratory noises during sleep or exercise were also common in our study (70\%), but none of the dogs exhibited stridor during rest, which was present in $66 \%$ of dogs referred for surgery (Roedler et al 2013). Mild sleep disturbances were also reported in our study (10\%), but sleeping problems were neither as common nor as severe as reported by Roedler et al (2013), where half of the dogs experienced considerable sleep disturbances. By comparing the owner questionnaire 
Table 4 Results of the 6-min and I,000-m walk tests for French Bulldogs, Pugs and control dogs presented as means $( \pm$ SD) and range.

\begin{tabular}{|c|c|c|c|c|c|c|}
\hline & BOAS- FBs & & BOAS+ FBs & BOAS- Pugs & BOAS+ Pugs & Control dogs \\
\hline \multirow[t]{3}{*}{ 6MWD (m) } & $638( \pm 49)$ & & $592( \pm 73)$ & $592( \pm 36)$ & $524( \pm 69)$ & $678( \pm 74)$ \\
\hline & $(575-745)$ & & $(435-695)$ & $(505-680)$ & $(400-700)$ & $(550-920)$ \\
\hline & $\mathrm{n}=27$ & $P=0.066$ & $\mathrm{n}=12$ & $P=0.001$ & $\mathrm{n}=18$ & $n=19$ \\
\hline \multirow[t]{3}{*}{$\mathrm{I}, 000 \mathrm{~m}(\mathrm{~min})$} & $9.44( \pm 0.64)$ & & $10.72( \pm 1.25)$ & $10.15( \pm 0.68)$ & $12.28( \pm 2.19)$ & $8.81( \pm 0.81)$ \\
\hline & $(8.15-10.77)$ & & $(8.42-12.15)$ & $(8.99-11.94)$ & $(9.06-17.55)$ & $(6.68-10.42)$ \\
\hline & $n=26$ & $P=0.010$ & $\mathrm{n}=10$ & $P=0.001$ & $\mathrm{n}=17$ & $n=19$ \\
\hline \multicolumn{7}{|l|}{$1,000-m$ recovery $(n)$} \\
\hline$\leq 5 \min$ & $4 / 26$ & & $0 / 10$ & $8 / 30$ & $0 / 17$ & $17 / 19$ \\
\hline$\leq 10 \mathrm{~min}$ & $14 / 26$ & & $4 / 10$ & $17 / 30$ & $8 / 17$ & $2 / 19$ \\
\hline$\leq 15 \mathrm{~min}$ & $7 / 26$ & & $2 / 10$ & $5 / 30$ & $5 / 17$ & $0 / 19$ \\
\hline$\geq 15 \mathrm{~min}$ & $1 / 26$ & $P=0.02$ & $4 / 10$ & $P=0.001$ & $4 / 17$ & $0 / 19$ \\
\hline $\begin{array}{l}\text { Combined } 1,000-\mathrm{m} \\
\text { time in } \leq 1 \mathrm{I} \text { min } \\
\text { and recovery } \\
\leq 15 \min ^{*}(\mathrm{n})\end{array}$ & $25 / 26$ & & $3 / 14 * *$ & $27 / 30$ & $5 / 18 * *$ & $19 / 19$ \\
\hline \multicolumn{7}{|c|}{$\begin{array}{l}\text { 6MWD: 6-minute walk distance; FB: French Bulldog; BOAS: brachycephalic obstructive airway syndrome; } \\
\text { BOAS- = no or mild signs, BOAS+ = moderate or severe signs; } \\
\text { P-values are presented between BOAS+ and BOAS- groups in each brachycephalic breed; } \\
\text { * Requirements for passing the Pug stress test for breeding by the German Pug Club; }\end{array}$} \\
\hline
\end{tabular}

Figure 3
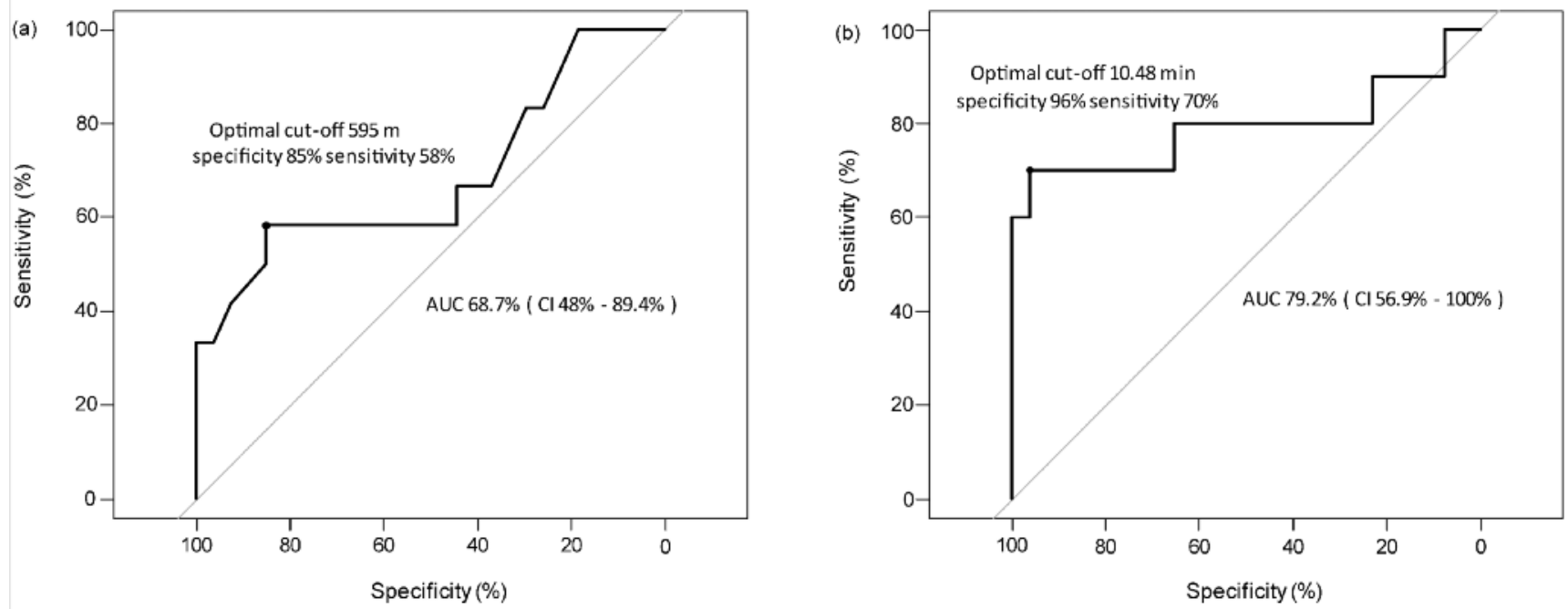

Receiver-operating characteristic (ROC) curves for the (a) 6-min walking distance and (b) I,000-m time for determining brachycephalic obstructive airway syndrome in BOAS+ and BOAS- French Bulldogs. 
Figure 4
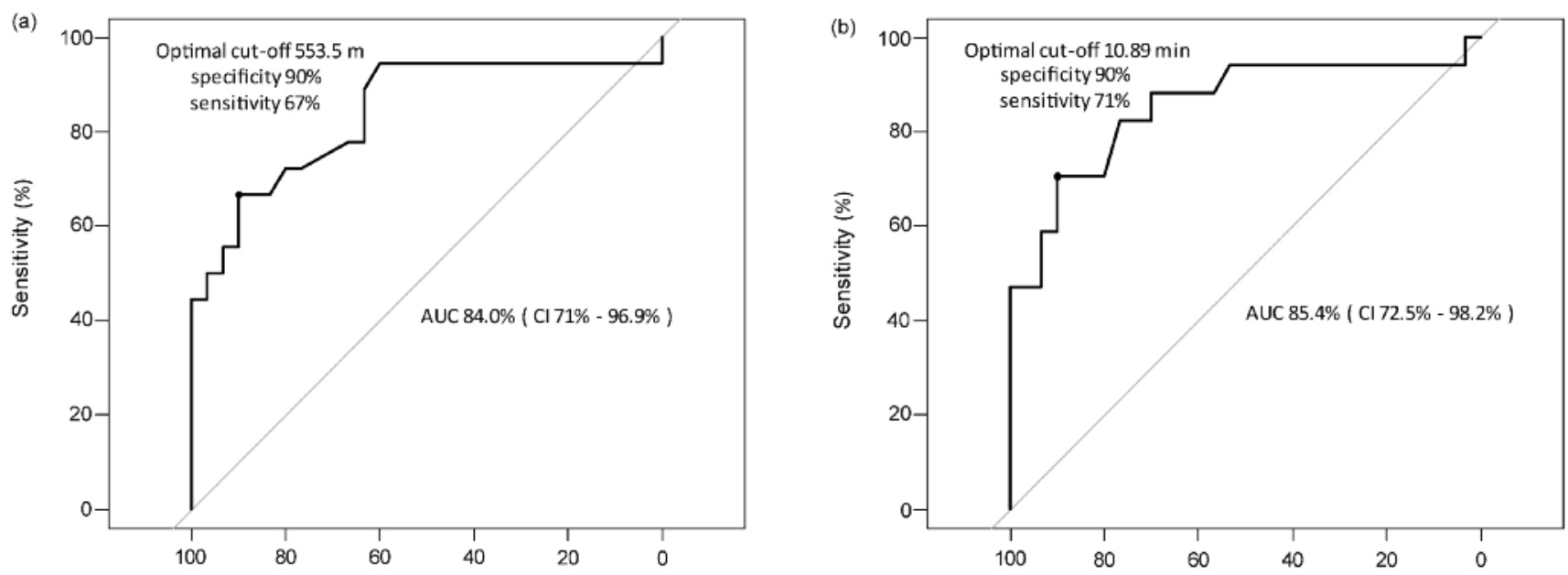

Receiver-operating characteristic (ROC) curves for the (a) 6-min walking distance and (b) 1,000-m time for determining brachycephalic obstructive airway syndrome in BOAS+ and BOAS- Pugs.

Table 5 ANOVA analyses* for association of severity of brachycephalic obstructive airway syndrome signs and 6-min walking distance and I,000-m time in French Bulldogs and Pugs.

\begin{tabular}{|c|c|c|c|c|}
\hline & BOAS 0 & BOAS I & BOAS 2 & BOAS3 \\
\hline $\begin{array}{l}\text { 6MWD (m) } \\
\text { LSM (95\% Cl) }\end{array}$ & $\begin{array}{l}612.25 \\
(579.00,645.50) \\
n=10\end{array}$ & $\begin{array}{l}612.02 \\
(596.14,627.90) \\
n=47\end{array}$ & $\begin{array}{l}566.99 \\
(542.15,591.82) \\
n=19\end{array}$ & $\begin{array}{l}532.54 \\
(499.72,565.37) ; \\
n=I I\end{array}$ \\
\hline \multicolumn{5}{|l|}{ Comparison } \\
\hline $\begin{array}{l}\text { BOAS I vs } \\
(95 \% \mathrm{Cl}) \\
P \text {-value }\end{array}$ & $\begin{array}{l}-0.23 \\
(-37.10,36.64) \\
0.99\end{array}$ & & & \\
\hline $\begin{array}{l}\text { BOAS } 2 \text { vs } \\
(95 \% \mathrm{CI}) \\
P \text {-value }\end{array}$ & $\begin{array}{l}-45.26 \\
(-86.77,-3.74) \\
0.03\end{array}$ & $\begin{array}{l}-45.03 \\
(-75.05,-15.02) \\
0.004\end{array}$ & & \\
\hline $\begin{array}{l}\text { BOAS } 3 \text { vs } \\
(95 \% \mathrm{Cl}) \\
P \text {-value }\end{array}$ & $\begin{array}{l}-79.70 \\
(-126.33,-33.07) \\
0.001\end{array}$ & $\begin{array}{l}-79.47 \\
(-116.52,-42.43) \\
<0.0001\end{array}$ & $\begin{array}{l}-34.44 \\
(-75.43,6.54) \\
0.10\end{array}$ & \\
\hline $\begin{array}{l}\text { I,000-m test time }(\mathrm{min}) \\
\text { LSM }(95 \% \mathrm{Cl})\end{array}$ & $\begin{array}{l}9.78 \\
(9.13,10.43) \\
n=10\end{array}$ & $\begin{array}{l}9.85 \\
(9.54,10.16) \\
n=46\end{array}$ & $\begin{array}{l}10.90 \\
(10.40,11.40) \\
n=18\end{array}$ & $\begin{array}{l}13.23 \\
(\mid 2.52,13.94) \\
n=9\end{array}$ \\
\hline \multicolumn{5}{|l|}{ Comparison } \\
\hline $\begin{array}{l}\text { BOAS I vs } \\
(95 \% \mathrm{Cl}) \\
P \text {-value }\end{array}$ & $\begin{array}{l}0.07 \\
(-0.66,0.79) \\
0.85\end{array}$ & & & \\
\hline $\begin{array}{l}\text { BOAS } 2 \text { vs } \\
95 \% \mathrm{Cl}) \\
P \text {-value }\end{array}$ & $\begin{array}{l}1.12 \\
(0.29,1.94) \\
0.008\end{array}$ & $\begin{array}{l}1.05 \\
(0.45,1.65) \\
0.0008\end{array}$ & & \\
\hline $\begin{array}{l}\text { BOAS } 3 \text { vs } \\
(95 \% \mathrm{Cl}) \\
P \text {-value }\end{array}$ & $\begin{array}{l}3.45 \\
(2.49,4.42) \\
<0.0001\end{array}$ & $\begin{array}{l}3.38 \\
(2.59,4.17) \\
<0.0001\end{array}$ & $\begin{array}{l}2.34 \\
(1.47,3.20) \\
<0.0001\end{array}$ & \\
\hline
\end{tabular}

* Age and weight were included as covariates;

BOAS: brachycephalic obstructive airway syndrome (grade $0=$ no, grade I = mild, grade $2=$ moderate, grade $3=$ severe signs); 6MWD: 6-min walking distance; LSM: least square mean; $\mathrm{Cl}$ : confidence interval. 
results of these different populations of French Bulldogs and Pugs, it is evident that BOAS signs do not restrict the lives of all brachycephalic dogs but, when severe, they are lifethreatening and constitute a major welfare problem. It should also be noted that in studies reporting owner-perceived BOAS signs of surgical cases (Torrez \& Hunt 2006; Riecks et al 2007; De Lorenzi et al 2009; Roedler et al 2013), owners are likely to be more perceptive with regard to BOAS signs due to their severity. Hence, the prevalence of daily BOAS signs may be underestimated in our population. However, the majority of French Bulldogs and Pugs in our study were considered to have no or mild BOAS signs also according to the veterinary evaluation.

An ideal tool for phenotype-based breeding selection should be easy to apply, objective and economical. Easily measurable external conformational risk factors associated with BOAS in Pugs, French Bulldogs and English Bulldogs were evaluated in a recent study by Liu et al (2017). Nostril stenosis was found to be the best predictor of BOAS status in all three breeds. In their study, the prevalence of moderate to severe stenotic nostrils was $75.4 \%$ in French Bulldogs, $65.3 \%$ in Pugs and $44.2 \%$ in Bulldogs. Accordingly, in our study, the prevalence of moderate to severe nostril stenosis was also alarmingly high: $86 \%$ in French Bulldogs and $80 \%$ in Pugs, but there was no significant correlation with the BOAS severity status. The difference between the findings may be caused by the lack of variation evident in our study. It is also possible that populations in different countries vary in the level of nostril stenosis.

In our previous study (Lilja-Maula et al 2017), the 6MWT and 1,000-m walk test were shown to be useful in assessing BOAS severity in English Bulldogs. Using a 3-min trotting test and a 5-min walk test, Riggs et al (2017) demonstrated that the exercise challenge constitutes an important part of the BOAS evaluation. A walk test on a leash is easily organised by breed clubs, for example, and is already used in several countries (Dutch Kennel Club 2014; Bartels et al 2015; Finnish Kennel Club 2018). Although a fixed speed test, using a treadmill, for example (Bartels et al 2015), could entail a more standardised exercise challenge when compared to a self-paced walk test, familiarisation and availability may pose difficulties (Kittelson et al 1996). In addition, a submaximal, self-paced walk test on a leash is also a safer method when considering dogs with potential respiratory disease. Self-paced exercise tests are also preferred over maximal exercise tests in human patients with moderate to severe heart or lung disease, as walking is well tolerated and better reflects the exercise level for daily physical activities (The American Thoracic Society [ATS] 2006).

The diagnostic ability of the $6 \mathrm{MWD}$ and $1,000-\mathrm{m}$ time to differentiate between the BOAS+ and BOAS- groups was assessed by ROC curves, where the area under the ROC curve (AUC) reflects the overall accuracy of the test. Values between 0.7 and 0.8 are considered satisfactory, 0.8 to 0.9 excellent, and more than 0.9 outstanding (Hosmer \& Lemeshow 2000; Mandrekar 2010). For a breeding selection test, a high sensitivity (ie low false negative rate)
Table 6 Pre- and post-I,000-m exercise test body temperatures of French Bulldogs, Pugs and control dogs presented as means ( \pm SD) and range.

\begin{tabular}{llll}
\hline & Pre-test $\left({ }^{\circ} \mathbf{C}\right)$ & Post-exercise $\left({ }^{\circ} \mathbf{C}\right)$ \\
\hline French Bulldogs & $38.4( \pm 0.42)$ & $P<0.000 I$ & $39.0( \pm 0.5 \mathrm{I})$ \\
$(\mathrm{n}=32)$ & $(37.5-39.2)$ & & $(37.8-40.3)$ \\
Pugs & $38.1( \pm 0.54)$ & $P<0.000$ & $38.7( \pm 0.57)$ \\
$(\mathrm{n}=47)$ & $(36.3-39.1)$ & & $(37.6-39.9)$ \\
Control dogs & $38.4( \pm 0.38)$ & $P=0.026$ & $38.7( \pm 0.44)$ \\
$(\mathrm{n}=19)$ & $(37.9-39.2)$ & & $(37.9-39.3)$ \\
\hline
\end{tabular}

could be considered ideal, especially when the disease in question is very severe or even lethal. However, as no gold standard for BOAS diagnosis exists and the functional grading system used in our study is subjective, we decided not to prefer either sensitivity or specificity. Therefore, the ideal cut-off value was defined as the point where the sum of sensitivity and specificity reaches its maximum (ie to maximise the number of correct test results). Based on AUC values, the $1,000-\mathrm{m}$ test has slightly better resolution ability in both brachycephalic breeds. In addition, a significant difference in the $1,000-\mathrm{m}$ test time was observed between all BOAS groups except between the mild and no signs groups while, in addition to that, the 6MWD did not demonstrate a significant difference between the severe and moderate groups. It might be that exercise of a longer duration more clearly manifests the exercise intolerance associated with BOAS. Based on the ROC curves, the optimal cut-off value for the 1,000-m test in French Bulldogs was 10.48 min with sensitivity of 0.70 and specificity of 0.96 , and 10.89 min in Pugs with sensitivity of 0.71 and specificity of 0.94 . A reference range for the test results can also be calculated as two times the standard deviation of values in healthy individuals. Using this, the upper maximum time limit for the 1,000 -m test based on the values of the control dogs was 10.43 , which is in line with optimal cut-off values and the 11-min time limit already used by the German Pug club (2014). In addition to walking performance, evaluation of the post-exercise recovery period is an important part of the test. None of the control dogs needed more than 10 min to recover and the majority recovered in 5. Furthermore, it was seen that in both brachycephalic breeds, the BOAS- dogs recovered significantly better than those in the BOAS+ group. Therefore, the requirement to recover in $15 \mathrm{~min}$ is quite moderate. With a maximum time of $11 \mathrm{~min}$ in the 1,000-m test and $15 \mathrm{~min}$ recovery, almost all of the BOAS- French Bulldogs (96\%) and Pugs $(90 \%)$ passed the test. However, some of the BOAS+ French Bulldogs (21\%) and Pugs (28\%) were also able to pass the test. For the most part, these included dogs with moderate BOAS signs, but one Pug with severe signs was also able to pass the test. This emphasises that in addition to the walk test, overall clinical assessment of BOAS signs is important to guide responsible breeding decisions. Furthermore, walking and recovery time limits could be further tightened up which, however, would also 
increase the portion of BOAS- dogs failing the test. Altogether, around $30 \%$ of Pugs and French Bulldogs failed the 1,000-m test and would therefore be considered unsuitable for breeding purposes. Almost half of the dogs in our study had or have since been used for breeding (Koiranet database 2018) and, hence, our results reflect the real population used for breeding in Finland. In a study by Bartels et al (2015), 50\% of Pugs failed the treadmill stress test. However, the exercise test type was different in our study. Other factors besides BOAS, such as the dogs' daily exercise habits, physical condition, motivation and personality can also affect the walking test performance.

Since abnormally growing nasal conchae and stenotic cartilages prevent normal thermoregulation in brachycephalic breeds (Hendricks 1992; Koch et al 2003; Oechtering 2010; Schuenemann \& Oechtering 2014), it is important to follow changes in body temperature during the exercise test. In our previous study (Lilja-Maula et al 2017), the rise in body temperature during the test was significantly greater in the English Bulldogs than in the control dogs, and the same was also seen in the current study between both brachycephalic breeds and the control dogs. None of the latter exceeded a body temperature of $39.3^{\circ} \mathrm{C}$ during or after the test, whereas a few French Bulldogs and Pugs had near or over $40^{\circ} \mathrm{C}$ body temperatures. However, when compared to our previous study with English Bulldogs (Lilja-Maula et al 2017), the high body temperature before or after exercise did not seem to be such a major concern for French Bulldogs and Pugs.

The limitations of our study are the small sample size and, particularly, the low number of severe BOAS cases and the subjective assessment of BOAS signs. Although internal airways were not examined, we have previously reported that the clinical BOAS grading system that was used reflected the severity of the pharyngolaryngeal components of BOAS seen in English Bulldogs (LiljaMaula et al 2017). In addition, all of the dogs in our study were young, with the median age being 2.5 years for French Bulldogs and 3 years for Pugs. As the mean age for seeking veterinary care due to BOAS is three to four years (Monnet 2008), the clinical signs can be expected to worsen. It should therefore be emphasised to breeders and potential puppy owners that any BOAS assessment or exercise test provides information only regarding the dog's present status. Breeders should also be encouraged to examine their dogs when they are older as well, and not to use very young males for breeding.

\section{Animal welfare implications}

The popularity of brachycephalic breeds, as well as health problems associated with brachycephaly, most notably BOAS, have increased in recent decades (Oechtering 2010). Therefore, actions to guide more responsible and ethical breeding choices are needed. Based on our previous study of English Bulldogs (LiljaMaula et al 2017) and the present study of French Bulldogs and Pugs, the exercise tolerance tests are useful in assessing the severity of BOAS. These sub-maximal, self-paced walk tests are also non-invasive, easy to organise and safe for the dogs. As BOAS is a multifactorial syndrome, other components besides exercise tolerance and recovery should also be evaluated during a breeding test. The subjective evaluation of nostril stenosis with a four-point scale (Liu et al 2016) is quick and easy to perform and should therefore be conducted together with a physical BOAS assessment and exercise test. By combining information from the physical BOAS assessment, namely the functional BOAS scale, nostril stenosis and exercise capacity, the breeder has the opportunity to make responsible breeding decisions related to BOAS. Although BOAS signs severely reduce the welfare of affected dogs, not all brachycephalic dogs suffer from clinically relevant BOAS signs, as seen in our study. By influencing the breeding choices and avoiding the use of dogs with distinct BOAS signs, the harmful impacts of brachycephaly could be diminished and the quality of life of the offspring improved.

\section{Conclusion}

In conclusion, in both brachycephalic breeds - the French Bulldog and the Pug — the more severely affected dogs performed both exercise tests more poorly than those with no or mild BOAS signs. The longer exercise, namely the 1,000-m walk test, seemed slightly better at differentiating between BOAS+ and BOAS- dogs. The results of this study further support the use of exercise tests as part of the breeding selection assessment for brachycephalic dogs. In future, studies evaluating the long-term impacts of BOAS breeding selection tools, such as exercise tests, on the welfare of offspring are needed, as well as follow-up studies to observe how age affects the results.

\section{Acknowledgements}

This study was supported by grants from the Finnish Cultural Foundation and the Finnish Veterinary Foundation, together with funding from the Academy of Finland.

We warmly thank the breed clubs as well as all of the owners and their dogs for their participation. We are also grateful to Laura Parikka for her invaluable help as research co-ordinator, to Petra Jaakonsaari for technical assistance during this study, and to Jouni Junnila, MSc, from 4Pharma for his help with the data analysis.

\section{References}

ATS (American Thoracic Society) Committee on Proficiency Standards for Clinical Pulmonary Function Laboratories 2002 ATS statement. Guidelines for the sixminute walk test. American Journal of Respiratory and Critical Care Medicine 166: III-II7. https://doi.org/I0.II64/ ajrccm.166.I.at I I02

Bartels A, Martin V, Bidoli E, Steigmeier-Raith S, Brühschwein A, Reese S, Köstlin R and Erhard M 2015 Brachycephalic problems of Pugs relevant to animal welfare. Animal Welfare 24: 327-333. https://doi.org//0.7/20/09 627286.24.3.327 
Boddy KN, Roche BM, Schwartz DS, Nakayama T and Hamlin RL 2004 Evaluation of the six-minute walk test in dogs. American Journal of Veterinary Research 65: 311-313. https://doi.org// 0.2460/ajvr.2004.65.3 I I

De Lorenzi D, Bertoncello D and Drigo M 2009 Bronchial abnormalities found in a consecutive series of 40 brachycephalic dogs. Journal of the American Veterinary Medical Association 235(7): 835-840. https://doi.org// 0.2460/javma.235.7.835

Dutch Kennel Club 2014 Convenant Bulldog, breeding rules. https://www.houdenvanhonden.nl/contentassets/27de95b0774b4 730990cfae5b7c4c3e4/convenant_bulldog-breeding_rules.pdf

Emmerson T 2014 Brachycephalic obstructive airway syndrome: a growing problem. Journal of Small Animal Practice 55: 543544. https://doi.org/ I 0. I I I I/jsap. 12286

Finnish Kennel Club 2018 Guideline for walking test. https://www.kennelliitto.fi/kasvatus-ja-terveys/koiranterveys/koiran-terveystutkimukset/kavelytestissa-arvioidaankoiran-rasituksensietoa

Hendricks JC 1992 Brachycephalic airway syndrome. Veterinary Clinics of North America: Small Animal Practice 22(5): I I45-I I 53. https://doi.org/10.1016/S0195-5616(92)50306-0

Hendricks JC 2004 Brachycephalic airway syndrome. In: King LG (ed) Textbook of Respiratory Disease in Dogs and Cats PP 310 318. Elsevier: St Louis, Missouri, USA. https://doi.org/I0.1016 /B978-0-72 I 6-8706-3.50044-X

Hosmer DW and Lemeshow S 2000 Applied Logistic Regression, Second Edition PP 160-164. John Wiley and Sons: New York, NY, USA. https://doi.org//0.1002/047|722/46

Kittelson MD, Johnson LE and Pion PD 1996 Submaximal exercise testing using lactate threshold and venous oxygen tension as endpoints in normal dogs and in dogs with heart failure. Journal of Veterinary Internal Medicine 10: 21-27. https://doi.org/ 10.1 III/j.1939-1676.1996.tb02019.x

Koch DA, Arnold S, Hubler M and Montavon PM 2003 Brachycephalic syndrome in dogs. Compendium on Continuing Education for the Practising Veterinarian 25: 48-55

KoiraNet 2018 The Finnish Kennel Club Breeding database. https://jalostus.kennelliitto.fi/frmEtusivu.aspx?Lang=en\&R

Lilja-Maula L, Lappalainen AK, Hyytiäinen HK, Kuusela E, Kaimio M, Schildt K, Mölsä S, Morelius M and Rajamäki MM 2017 Comparison of submaximal exercise test results and severity of brachycephalic obstructive airway syndrome in the English Bulldog. The Veterinary Journal 219: 22-26. https://doi.org/10.1016/j.tvjl.2016.11.019

Lilja-Maula L, Laurila HP, Syrjä P, Lappalainen AK, Krafft E, Clercx C and Rajamäki MM 2014 Long-term outcome and use of 6-minute walk test in West Highland White Terriers with idiopathic pulmonary fibrosis. Journal of Veterinary Internal Medicine 28: 379-385. https://doi.org/ I0.1 I I I/jvim. I228 I

Liu N-C, Adams VJ, Kalmar L, Ladlow JF and Sargan DR 2016 Whole-body barometric plethysmography characterizes upper airway obstruction in 3 brachycephalic breeds of dogs. Journal of Veterinary Internal Medicine 30: 853-865. https://doi.org/I0.1 I I I/jvim. I 3933
Liu N-C, Sargan DR, Adams VJ and Ladlow JF 2015 Characterisation of brachycephalic obstructive airway syndrome in French Bulldogs using whole-body barometric plethysmography. PLoS ONE 10: e013074l. https://doi.org//0.137I /journal.pone.013074 I

Liu N-C, Troconis EL, Kalmar L, Price DJ, Wright HE, Adams VJ, Sargan DR and Ladlow 2017 Conformational risk factors of brachycephalic obstructive airway syndrome (BOAS) in Pugs, French Bulldogs, and bulldogs. PLoS ONE 12: e0181928. https://doi.org/10.1371/journal.pone.0181928

Mandrekar JN 2010 Receiver operating characteristic curve in diagnostic test assessment. Journal of Thoracic Oncology 5(9): I315I3 16. https://doi.org//0.1097/JTO.0b0 I 3e3 I8 lec I73d

Manens J, Ricci J, Damoiseaux C, Gault S, Contiero B, Diez $M$ and Clercx C 2014 Effect of bodyweight loss on cardiopulmonary function assessed by 6-Minute Walk Test and arterial blood gas analysis in obese dogs. Journal of Veterinary Internal Medicine 28: 37 I-378. https://doi.org/I 0. I I I I/jvim. I 2260

Monnet E 2008 Brachycephalic airway syndrome (CVC Proceedings). http://veterinarycalendar.dvm360.com/brachycephalic-airway-syndrome-proceedings-2

Oechtering GU 2010 Brachycephalic syndrome - new information on an old congenital disease. Veterinary Focus 20: 2-9

Packer RMA, Hendricks A and Burn CC 2012 Do dog owners perceive the clinical signs related to conformational inherited disorders as 'normal' for the breed? A potential constraint to improving canine welfare. Animal Welfare 21: 81-93. https://doi.org/I0.7I20/0962728I2XI3345905673809

Packer RMA, Hendricks A, Tivers MS and Burn C 2015 Impact of facial conformation on canine health: Brachycephalic Obstructive Airway Syndrome. PLoS ONE 10: e0137496. https://doi.org/10.137//journal.pone.0137496

Packer RMA and Tivers MS 2015 Review: Strategies for the management and prevention of conformation-related respiratory disorders in brachycephalic dogs. Veterinary Medicine: Research and Reports 6: 219-232. https://doi.org//0.2I47/VMRR.S60475

Poncet CM, Dupré GP, Freiche VG, Estrada MM, Poubanne YA and Bouvy BM 2005 Prevalence of gastrointestinal tract lesions in 73 brachycephalic dogs with upper respiratory syndrome. Journal of Small Animal Practice 46: 273-279. https://doi.org// 0.1 I I I/j. I748-5827.2005.tb00320.x

Riecks TW, Birchard SJ and Stephens JA 2007 Surgical correction of brachycephalic syndrome in dogs: 62 cases (19912004). Journal of the American Veterinary Medical Association 230(9): 1324- | 328. https://doi.org//0.2460/javma.230.9.1324

Riggs J, Liu N-C and Ladlow JF 2017 A comparison of two different types of exercise test for the investigation and clinical grading of upper airway disease severity in brachycephalic dogs. 26th European College of Veterinary Surgeons (ECVS) Annual Scientific Meeting. I3-I5 July 2017, Edinburgh, UK

Roedler FS, Pohl S and Oechtering GU 2013 How does severe brachycephaly affect dogs' lives? Results of a structured preoperative owner questionnaire. The Veterinary Journal 198: 606-610. https://doi.org//0.1016/j.tvjl.2013.09.009 
Schuenemann RS and Oechtering GU 2014 Inside the brachycephalic nose: Intranasal mucosal contact points. The Journal of the American Animal Hospital Association 50(3): I49-I58. https://doi.org//0.5326/JAAHA-MS-599|

Swimmer RA and Rozanski EA 201I Evaluation of the 6minute walk test in pet dogs. Journal of Veterinary Internal Medicine 25: 405-406. https://doi.org/I0.1 I I I/j.1939-1676.20I I.0689.x

The German Pug Club 2014 Belastungstest für Hunde kurzschnäuziger Rassen. https://www.vdh.de/news/artikel/belastungstest-fuer-hunde-kurzschnaeuziger-rassen/. [Title translation: Stress test for short-nosed dog breeds]
The Kennel Club 2017 French Bulldogs Set to Overtake Labradors As UK's Most Popular Dog Breed. https://www.thekennelclub.org.uk/pressreleases/20I7/february-20 I/french-bulldogs-set-to-overtakelabradors-as-uk-s-most-popular-dog-breed/

The Kennel Club 2018 Breed Registration Statistics. https://www.thekennelclub.org.uk/media/I I 5944I/top_20_breeds _2016_-_2017.pdf

Torrez CV and Hunt GB 2006 Results of surgical correction of abnormalities associated with brachycephalic airway obstruction syndrome in dogs in Australia. Journal of Small Animal Practice 47: I50-I54. https://doi.org// 0. I I I I/j. I 748-5827.2006.00059.x 